\title{
Evaluación de conocimientos y habilidades en el areá de Geografía en estudiantes de 8vo. Básico y 4to. Medio'
}

\author{
Joaquín Gallastegui-Vega2, Javier Vergara-Núñez³, Ignacio Rojas-Rubio4, \\ Juan Gálea-Alarcón ${ }^{5}$ y Juan Gonzalo Saldaña-Álvarez ${ }^{6}$
}

\section{RESUMEN}

La investigación tuvo por objetivo evaluar el desarrollo de la disciplina de geografía respecto de conocimientos y habilidades fundamentales a alcanzar en estudiantes de $8 \mathrm{vo}$. Básico y 4to. Medio. El estudio curricular constata la subvaloración de la disciplina en comparación con el área de historia lo cual, según la literatura revisada, permite deducir que las propuestas temático-programáticas están desactualizadas y los diseños de aprendizaje descontextualizados. Se evaluó estudiantes de establecimientos educacionales municipales y subvencionados de Valparaíso, Viña del Mar y San Felipe $(n=428)$, tras elaborar, validar y aplicar un instrumento focalizado en el área cognitiva del conocimiento y comprensión de contenidos, análisis de situaciones simuladas y aplicación de conocimientos fundamentales contextualizados, además de expresar el grado de seguridad en la respuesta, denota niveles de conocimiento sólo aceptables (Acierto total=32\%) y bajos índices de seguridad en las respuestas. Los aciertos se focalizan en preguntas contextualizadas espacial o temporalmente.

Palabras clave: Disciplina de geografía, conocimientos fundamentales de geografía, desarrollo de habilidades fundamentales de geografía.

\begin{abstract}
The objective of the research was to evaluate the development of the discipline of geography with respect to fundamental knowledge and skills to be attained in 8th grade students. Basic and 4th. Middle. The curricular study confirms the undervaluation of the discipline in comparison to the area of history which, according to the reviewed literature, allows to deduce that the thematic-programmatic proposals are outdated and the learning designs decontextualized. We evaluated students from municipal and subsidized educational institutions in Valparaíso, Viña del Mar and San Felipe $(n=428)$, after elaborating, validating and applying an instrument focused on the cognitive area of knowledge and understanding of contents, simulated situations analysis and application Of contextualized fundamental knowledge, in addition to expressing the degree of security in the response, denoted only acceptable levels of knowledge (Total success = $32 \%$ ) and low security indexes in the responses. The answers focus on contextualized questions spatially or temporally.
\end{abstract}

Keywords: Discipline of geography, basic knowledge of geography, development of basic geography skills.

Este artículo es producto de la investigación enmarcada en el Convenio de Desempeño en Formación de Profesores 1203 desarrollado entre la Universidad de Playa Ancha y el Ministerio de Educación (2013- 2015) y del proyecto regular de investigación DIGI (HUM 03 16-17) de la Universidad de Playa Ancha. Artículo recibido el 30 de julio de 2016, aceptado el 30 de enero de 2017 y corregido el 28 de julio de 2017.

Departamento Disciplinario de Historia, Universidad de Playa Ancha (Chile). E-mail: jgallast@upla.cl

Departamento de Educación y Pedagogía, Universidad de Playa Ancha (Chile). E-mail: jvergara@upla.cl

Departamento Disciplinario de Historia, Universidad de Playa Ancha (Chile).E-mail: ignacio.rojas@upla.cl

Departamento Disciplinario de Historia, Universidad de Playa Ancha (Chile). E-mail: jgalea@upla.cl

Departamento Disciplinario de Historia, Universidad de Playa Ancha (Chile). E-mail: juan.saldana@upla.cl 
Este artículo da cuenta de los resultados obtenidos tras el desarrollo de una investigación focalizada en una evaluación de los aprendizajes del campo conceptual y procedimental (Coll et al., 1994) en el área de Geografía, cuya finalidad es aportar antecedentes respecto de los aprendizajes y desarrollo de habilidades efectivamente alcanzados por los estudiantes al cierre del ciclo básico y medio del sistema regular de estudios, en una muestra de 428 estudiantes. La investigación comprendió tres momentos: (1) revisión de la literatura científica con el objeto de fundar un referente de conocimientos actualizados que sustentara el desarrollo de las fases posteriores, (2) análisis curricular de los Programas de Estudio del área de la geografía en cuyos resultados se funda el diseño del instrumento de evaluación que se emplea y, (3) elaboración, prueba y aplicación del test de conocimientos y habilidades fundamentales en geografía.

“La Universidad de Playa Ancha, pedagógica en su génesis, se encuentra comprometida en la actualidad con el desafío de innovar en la formación de profesionales docentes, actualizando el diseño curricular de la formación, introduciendo nuevos conceptos didácticos y evaluativos ajustados al diseño de demostración de competencias y centrado en los aprendizajes de los estudiantes" (UPLA, 2013, a y b), y la incorporación de múltiples recursos de apoyo a los aprendizajes. Es en este contexto de renovación formativa, donde emerge la necesidad de que cada una de las líneas columnares de la formación revisen igualmente sus diseños: Formación Profesional-pedagógica; Formación en la Práctica, Formación en el Sello de la Universidad y Formación Disciplinar. Justamente, en esta última línea formativa es donde se sitúa esta investigación, buscando aportar antecedentes sobre los aprendizajes efectivamente alcanzados por los estudiantes al cierre de los ciclos básico y medio del sistema regular de estudio, punto desde el cual debiera revisitarse todo cuanto constituye la formación de los futuros Profesores de Geografía en la Universidad.

El desarrollo atiende a los tres focos de estudio indicados; primero se da cuenta de la problemática y sus antecedentes; seguidamente se presentan los resultados de la revisión de la literatura científica y cuanto concierne al análisis de los referentes teóricos de la disciplina, estableciendo un cuerpo conceptual que permita primeramente desarrollar el estudio curricular del Programa Oficial de la Asignatura Geografía y, tras ello, establecer una base teórica que funde las decisiones de elaboración del instrumento de evaluación que se emplea con la muestra de estudiantes evaluados. Seguidamente, se procede con el segundo foco de la investigación dado por el análisis curricular del Programa oficial de la asignatura, instancia de estudio de la cual emergen las bases temáticas para desarrollar la construcción del instrumento que, finalmente cierra con la entrega de los resultados obtenidos del estudio de campo. Por último, se discute los resultados, se señalan las conclusiones y se da cuenta de las proyecciones de la investigación en completo.

\section{Planteamiento del problema en estudio y sus antecedentes}

Un estudio recientemente efectuado en la Universidad de Playa Ancha, en una muestra significativa de estudiantes del sistema regular de educación (Castillo y Jaime, 2012), da cuenta de que las aprehensiones docentes tienen asidero. Los autores mencionados logran demostrar que los programas de estudio del área Geográfica tienen una menor amplitud temática ${ }^{7}$, tras aplicar un test a la muestra estudiada (similar en atributos a la de esta investigación), los resultados alcan-

Ver en http://www.curriculumenlineamineduc.cl 
zados denotan un desarrollo cognitivo muy bajo, tanto a nivel de conocimientos esenciales como respecto de algunas habilidades básicas del área.

Desde la descripción del contexto en que se ubica el estudio, se delimita la problemática en torno a cinco elementos distintivos: (1) una subvaloración de la disciplina geográfica, expresada en el poco tiempo dispuesto para desarrollarla y el escaso desarrollo curricular en el programa mismo, (2) la existencia de propuestas temático-programáticas desactualizadas del entorno natural, social y cultural de los estudiantes, (3) diseños de aprendizaje que no consideran cuestiones claves como el contexto en donde se producen los aprendizajes, asociado a (4) una didáctica docente poco pertinente $y$, como consecuencia, (5) aprendizajes descendidos en los estudiantes. Estos cinco elementos distintivos están directamente relacionados con el campo de investigación que se ha establecido explorar.

A partir de lo antes señalado, se busca responder las siguientes preguntas de investigación:

a. ¿Cómo se estructuran los Programas de Estudio respecto de los referentes teóricos que orientan en la actualidad a la disciplina? ¿Cómo recogen estos Programas de estudio los lineamientos de la teoría geográfica actual? ¿Qué elementos referidos a contenidos y habilidades proponen los Programas y como éstos se distrubuyen a lo largo de la formación?

b. ¿Qué niveles de logro de conocimientos y desarrollo de habilidades básicas alcanzan?

En función de estos antecedentes y de la necesidad de revalorizar la disciplina, se estima necesario profundizar sobre esta realidad educativa y enriquecer el debate sobre el rol curricular que posee la geografía; se busca cruzar la reflexión epistemológica sobre la disciplina de estudio con la experiencia en docencia que tienen los investigadores atendiendo la calidad de profesores del área en el sistema educativo regular, y tras la evaluación, se pretende observar el contexto a la luz de los resultados que reporta el empleo de un instrumento estandarizado.

\section{Los antecedentes que emergen de la base epistemológica de la disciplina}

En este apartado se dará cuenta de dos cuestiones esenciales para referenciar posteriormente el análisis curricular, la elaboración del instrumento evaluativo y al final como criterio de análisis en la discusión de los resultados. En primer lugar, una revisión de la teoría de la geografía y la determinación de los conceptos clave; tras ello, un análisis de la geografía como disciplina de estudio, revisión que permite ponerla en la perspectiva del estudio de campo del que se da cuenta más adelante.

\section{Teoría de la geografía contemporánea y determinación de conceptos clave para su enseñanza}

Como la mayoría de las disciplinas, la Geografía presenta una serie de estudios y objetivos múltiples y variados según sea el paradigma que se emplee, aunque puede intentarse detectar rasgos comunes y elementos de continuidad entre todos ellos (Rodríguez, 2010). Es la noción de "espacio" la que une e integra bajo un prisma común las diferentes corrientes actuales, pero ade- 
más de la denominada "conciencia espacial" por parte de las sociedades humanas. Es muy difícil y complejo llegar a una definición única del constructo "espacio geográfico", ya que su racionalización ha sido histórica, por su evolución y sus diferentes acepciones según la realidad observada, ya sea material y social, del desarrollo de la reflexión teórica y de los diferentes sustratos como es el ideológico de quienes intentan definirlo. En otras palabras, la realidad material, la ideología y la reflexión teórica ha variado a través del tiempo. Cabe destacar que la Geografía, en la actualidad no tiene monopolio de lo espacial, dado que en las Ciencias Sociales existe una constante reconceptualización que está reivindicando el factor espacio en lo social, produciéndose así una relación interdisciplinar (Méndez, 1995; MINEDUC 2009).

Según Méndez (1995), los Geógrafos han tendido a organizar su pensamiento en torno a cinco grandes temas: (1) La diferencia espacial, siendo la región el concepto básico, (2) El paisaje (natural o cultural), (3) La relación hombre-entorno, es decir, la Geografía como ecología humana; (4) La distribución espacial, describiendo y explicando la localización de los fenómenos de la superficie terrestre (análisis locacional), y (5) Análisis locacional (relacionado con lo anterior) desde el punto de vista geométrico, según los enfoques teorético-cuantitativo.

Las cinco temáticas definen un campo amplio de estudio, que va desde el espacio como simple marco o escenario pasivo donde se sitúan los objetos, es decir, el espacio euclidiano, absoluto, definido por unas coordenadas precisas con existencia propia e independiente de la materia, estrechamente vinculado a la Cartografía (espacio como continente); hasta el espacio que se define por sobre todo por sus propiedades o contenidos específicos (espacio contenido), donde la localización cuenta tan solo como un agregado complejo. Es el espacio social, con sus simbolismos y sus capacidades tecnológicas y productivas, así como sus valores culturales e ideológicos dominantes, donde el espacio Geográfico es el producto social.

El espacio como producto social es un objeto complejo y polifacético, es lo que materialmente la sociedad crea y recrea, con una entidad física definida; es una representación social y es un proyecto, en el que operan individuos, grupos sociales, instituciones, relaciones sociales, con sus propias representaciones y proyectos. El espacio se presenta, además, a través de un discurso socialmente construido, que mediatiza al tiempo que vehicula la representación y prácticas sociales, es decir, este espacio tiene una doble dimensión: es a la vez material y representación mental, objeto físico y objeto mental (Gallastegui y Gálea 2009).

Por su parte, Santos (2000) sostiene que toda disciplina se define por el objeto estudiado y lo que estudia la geografía es la "espacialidad de los fenómenos". De ahí que como punto de partida Santos propone que el espacio sea definido como un conjunto indisoluble de sistemas de objetos (lo físico) y sistema de acciones (lo social), flujos y fijos. Para Lindon (2008), los cambios que ha experimentado la Geografía, están asociados a los denominados giros culturales de la posmodernidad que hace referencia a la ausencia de lo espacial en la etapa de la modernidad, es decir, se ha omitido la "especialidad de lo social", en cambio ha tenido gran importancia la dimensión temporal, cuando en realidad son indisociables ambas dimensiones (tiempo-espacio). Cabe reconocer que en la modernidad la geografía se ha acercado poco a la Teoría Social lo cual se debe, en especial, a la materialidad que se le ha asignado al espacio (espacio como continente). Hiernaux y Lindon (2006) se refieren a la importancia de "lo espacial" en los problemas sociales reconociendo al siglo XXI como un siglo espacial. 
Considerando los elementos anteriormente planteados, desde el manejo conceptual que el estudiante debería tener, según el mapa de progreso correspondiente al desarrollo de la disciplina en geografía y los conceptos expuestos anteriormente, el aprendizaje se sustenta en tres dimensiones, las cuales se desarrollan de forma interrelacionada:

- Localización espacial y comprensión sistémica del espacio geográfico: Se refiere al conocimiento de la ubicación y distribución espacial de elementos y procesos geográficos y a la comprensión de la dinámica espacial de un territorio determinado, integrando diversas variables naturales, sociales, económicas, políticas y culturales. Esta dimensión progresa desde una visión geográfica general de la Tierra y de la identificación de relaciones simples entre elementos geográficos, hacia un conocimiento más detallado y sistémico del planeta y un entendimiento de la interrelación entre diversas variables en la configuración del espacio geográfico.

- Habilidades de análisis del espacio geográfico: Apunta al desarrollo de las habilidades relacionadas con la observación e interpretación del espacio geográfico directamente o a través de distintas fuentes de información, aplicando categorías geográficas cada vez más complejas, para analizar problemas geográficos relevantes, formulando hipótesis sobre sus causas y su impacto territorial.

- Valoración y actuación responsable con el espacio geográfico: Enfatiza el desarrollo de actitudes de cuidado y responsabilidad con el espacio que habitan a escala local y global, comprendiendo su rol en la sustentabilidad ambiental y en la valoración de los lugares de pertenencia.

\section{La situación actual de la geografía como disciplina de estudio}

Ya se mencionaba la importancia conceptual del espacio social en el desarrollo del conocimiento escolar, en términos simbólicos, capacidades tecnológicas y productivas, valores culturales e ideológicos, conceptualización que no ha tenido un desarrollo consecuente en nuestro sistema escolar, lo que dificulta a estudiantes y a profesores el manejo de conceptos clave como los mencionados: la región, el paisaje, la relación hombre-medio, distribución espacial, análisis locacional. Es importante considerar que muchos de estos conceptos ya no son trabajados en el área disciplinar de la Historia y la Geografía, sino en las asignaturas de Física o Comprensión del Medio Natural y Social, las cuales se insertan en el área de las Ciencias Naturales y Exactas. A esto se suma que, a nivel de la formación inicial de éstos profesores (Física, Química y/o Ciencias Naturales), que imparten estos contenidos, carecen de las competencias disciplinares necesarias para la enseñanza de la Geografía (Gallastegui et al., 2014), pues en su formación no se consideran los contenidos especificos de la disciplina.

Esta suma de elementos da como resultado una acumulación de conceptos, hechos y datos que el alumnado debe memorizar sin comprender, no sólo en su sentido preciso, sino además en las relaciones que les presentan los temas de un libro de texto. Por mucho tiempo ha imperado en el sistema una didáctica centrada en la aplicación de técnicas y métodos para el aula, el recetario para las buenas prácticas pedagógicas y un conjunto de recursos para el aprendizaje de contenidos (recepción, memorización y reproducción). Se puede resumir la situación actual de la Geografía en aspectos como los siguientes: 
- En las bases curriculares (MINEDUC, 2009), la Geografía como disciplina de estudio aparece en situación subestimada frente a la disciplina de Historia; esta circunstancia no le permite cumplir de un modo cabal su rol formativo en el alumno, ni desarrollar plenamente su objeto como disciplina distinta. La relevancia que se le asigna en el programa difiere en el tiempo que se dispone para su tratamiento (unidades de aprendizaje) y, consecuentemente, la profundidad con que se les debe desarrollar.

- La distribución de los diversas unidades de contenido de la Geografía no se adecuan al contexto de aprendizaje de los estudiantes ni a las exigencias modernas del proceso de enseñanza- aprendizaje. Esto último, según Méndez (2008), se debe a que la Geografía ha experimentado un rápido crecimiento y una cierta fragmentación en las últimas décadas que dificultan el mantenimiento de una imagen integrada y la trasmisión de nuevas temáticas y teorías desde el ámbito de la investigación de la enseñanza. Generalmente, los contenidos no son selectivos en cuanto a centralizar los problemas en torno a materias de interés para los alumnos y en unidades que partan de lo local y concreto. En torno a lo mismo, la literatura científica confirma que tanto la valoración del contexto en donde se encuentra la escuela -y el propio estudiante-, como las experiencias previas del estudiante son elementos didácticos esenciales que, al ser incorporados al diseño como a la práctica misma del aula, facilitan los aprendizajes de los estudiantes (Novak, 1998; Kemmis, 1993; Ausubel, 1987; Gimeno y Pérez, 1983).

- La sola observación de los programas de estudio lleva a constatar que hay un notable desequilibrio entre los contenidos de Geografía Humana y los de Geografía Física, siendo mayoritarios estos últimos. Este desequilibrio se puede ejemplificar con la estructura presentada en textos de divulgación pedagogica ${ }^{8}$ en el que de la seis unidades que están relacionadas con Geografía, cuatro de ellas presentan solo rasgos físicos (66\%), además de presentar una visión descriptiva.

- En relación con lo señalado en el punto anterior, se constata que los programas, en general, se muestran desactualizados en el tratamiento de los contenidos, evidenciando la necesidad de revisión y actualización, de modo que reflejen los requerimientos del mundo actual, como para lograr atender las problemáticas de países en desarrollo como Chile. Temas como la responsabilidad social (nueva ciudadania) ante las temáticas medioambientales, el uso responsable de la energía, la vinculación con el medio geográfico, entre otros, no están abordados convenientemente en los programas.

- Como se ha señalado al comienzo, se observa en las aulas que la enseñanza de la Geografía se caracteriza por un predominio en el uso de métodos didácticos expositivos y librescos que tienden al verbalismo; una didáctica orientada a la acumulación de conocimientos, con alto predominio y excesiva memorización.

- La Geografía se enseña con criterio excesivamente analítico y disgregante, en temas aislados y rígidos. Lo cual va en sentido contrario del que indican los lineamientos internaciona-

Se consultaron textos escolares de apoyo a la docencia en el área: el libro de Primero Medio "Historia y Geografía" del año 2000 de la Editorial Mare Nostrum. 
les sobre educación geográfica que, según Prats (2011), han buscado cambiar el enfoque de la enseñanza de la Geografía la que debe adecuarse a los "nuevos contextos sociales e internacionales".

Si bien, los aspectos que se han señalado abarcan distintos ámbitos del quehacer pedagógico, análisis y diseño curricular, didáctica, entre otros, con ellos se evidencia que las prácticas del aula se desarrollan de manera descontextualizada de la realidad y de la cotidianidad del estudiante. La disciplina geográfica aparece como un conjunto de conocimientos poco valorados y restringidos por el propio docente ya que muchos de los aprendizajes están basados en el dominio espacio temporal y, en consecuencia, el aprendizaje por parte de los estudiantes requiere de un esfuerzo superlativo, sobre todo al considerar que la clase de Geografía se centra en el mapa, el cual aparece mal utilizado (Liceras, 2000).

¿Qué se debe enseñar en Geografía? y ¿qué es lo que realmente aprenden los estudiantes del sistema en Geografía? Son preguntas que no deben estar ausentes de toda reflexión pedagógica, más aún por las serias implicancias en la formación ciudadana de los estudiantes y en la construcción epistémica del conocimiento geográfico.

La enseñanza de la Geografía debe considerar no sólo los avances en la construcción del conocimiento geográfico, sino también el modelo curricular y didáctico con el que se desea enseñar la disciplina. Reflexionar el currículo en Ciencias Sociales (Geografía) implica tomar postura y asumir la responsabilidad y compromiso de enseñar con el fin de suscitar un aprendizaje en el otro. Implica abandonar el estado de comodidad y acomodaticio de la pseudo neutralidad científica del "paradigma empírico racional" que es un pasaje gratuito a las prácticas pedagógicas descontextualizadas que se centran en la enseñanza del contenido por el contenido, propios de una agenda clásica-academicista que, en el actual contexto de la sociedad del conocimiento, la convierten en un relicto de la modernidad.

Desde esta mirada, la enseñanza de una Geografía, a partir de una didáctica pertinente, reflexiva y contextual, contribuye al logro de aprendizajes significativos y profundos en los estudiantes del sistema escolar. El modelo didáctico debe estar en congruencia con el modelo curricular y el modelo evaluativo (De Camilloni, 1996).

\section{Analisis curricular de los programas de estudio oficiales}

Posterior a la revisión de los elementos teóricos que orientan en la actualidad a la disciplina en su desarrollo como campo del conocimiento, y que fundan su transposición en el aula, se procedió al análisis de los Programas de Estudio para cada uno de los niveles educativos y para cada una de las asignaturas de estudio ${ }^{9}$, programas que son los documentos a través de los cuales el MINEDUC expresa la línea formativa nacional.

Atendiendo algunas normas metodológicas esenciales sobre el método de análisis documental hermenéutico, se procedió en primer término a la identificación de los documentos que serían 
tratados, constituyendo de esta forma el corpus de análisis; una vez compilados se procedió a analizar y a explicitar desde el contenido declarado los elementos que se incorporan en la disciplina. Se procuró definir los ámbitos del aprendizaje para cada nivel educativo, estableciendo los campos cognitivos que se declaran bajo formato de "Objetivos de Aprendizaje Esperado" para la disciplina de Geografía (Mucchielli, 2006; Bardin, 1996; Krippendorf, 1990; Miles \& Huberman 2003).

Para establecer el ordenamiento de los campos cognitivos se estableció una base conceptual que permitiera el análisis de los Programas de Estudio, para cuyo efecto se recurre a la definición que propone Coll et al. (1994) y Delors (1996) ${ }^{10}$; ambos autores sostienen una división de los aprendizajes separándoles en "cognitivos", "procedimentales" y "actitudinales- valóricos", lo cual tiene su expresión en los Programas nacionales como "conocimientos", "habilidades" y "actitudes". De acuerdo con esta clasificación, se procedió luego a seleccionar, extraer y categorizar desde los Programas aquellos objetivos que refieren los aprendizajes según su tipo. Atendiendo los objetivos de esta investigación, solo se consideró los aprendizajes esperados de los ambitos del conocimientos y las habilidades.

Una vez revisados los Programas de cada curso, se elaboró el Cuadro $N^{\circ} 1$ que organiza los aprendizajes según cada uno de los niveles de estudio.

\section{Cuadro $\mathrm{N}^{\circ} 1$}

Objetivos (conocimientos y habilidades) fundamentales de acuerdo a nivel de estudio y preguntas asociadas para construcción de: "Test de conocimientos generales de Geografía"

\begin{tabular}{|l|c|c|}
\hline Objetivos (conocimientos y habilidades) & $\begin{array}{c}\text { Nivel de } \\
\text { estudios } \\
\text { vinculado }\end{array}$ & $\begin{array}{c}\text { Preguntas } \\
\text { asociadas } \\
\text { a los objetivos }\end{array}$ \\
\hline $\begin{array}{l}\text { Conocimientos } \\
\text { Reconocer que los mapas y los planos son formas de } \\
\text { representar lugares. }\end{array}$ & \\
$\begin{array}{l}\text { Identificar a Chile en mapas, incluyendo la cordillera de los } \\
\text { Andes, el océano Pacífico y las ciudades. } \\
\text { Observar y describir paisajes de su entorno local, utilizando } \\
\text { vocabulario geográfico adecuado }\end{array}$ & $1^{\circ}, 2^{\circ}$ y $3^{\circ}$ \\
Básico & $\begin{array}{c}\text { Pregunta(s) } \\
\mathrm{N}^{\circ}:\end{array}$ \\
$\begin{array}{l}\text { Habilidades: } \\
\text { Localizar a Chile en mapamundis o globos terráqueos y } \\
\text { distinguir diferentes representaciones de la Tierra. }\end{array}$ & \\
\hline
\end{tabular}

Ambos autores figuran como referentes en los diseños curriculares nacionales (Marco Curricular Nacional, Programas de Estudio, entre otros) 
Continuación Cuadro $N^{\circ} 1$

\begin{tabular}{|c|c|c|}
\hline Objetivos (conocimientos y habilidades) & $\begin{array}{c}\text { Nivel de } \\
\text { estudios } \\
\text { vinculado }\end{array}$ & $\begin{array}{c}\text { Preguntas } \\
\text { asociadas } \\
\text { a los objetivos }\end{array}$ \\
\hline $\begin{array}{l}\text { Conocimientos: } \\
\text { - Clasificar y caracterizar algunos paisajes de Chile según su } \\
\text { ubicación en la zona norte, centro y sur del país, observando } \\
\text { imágenes, y utilizando diversas fuentes y un vocabulario } \\
\text { geográfico adecuado (océano, río, cordillera de los Andes y de } \\
\text { la Costa, desierto, valle, costa, volcán, archipiélago, isla, fiordo, } \\
\text { lago, ciudad y pueblo, entre otros). } \\
\text { - } \quad \text { Reconocer diversas expresiones del patrimonio natural de Chile } \\
\text { y de su región, como paisajes, flora y fauna característica, y } \\
\text { parques nacionales, entre otros. } \\
\text { Habilidades: } \\
\text { - Leer y comunicar información geográfica mediante distintas } \\
\text { herramientas (planos, mapas, globos terráqueos y diagramas). } \\
\text { - Orientarse en el espacio, utilizando categorías de ubicación } \\
\text { relativa (derecha, izquierda, lejos, cerca) y asociando referentes } \\
\text { locales (cordillera, cerro, mar y otros) a los puntos cardinales. }\end{array}$ & $\begin{array}{l}2^{\circ}, 3^{\circ} \\
\text { Básico }\end{array}$ & $\begin{array}{l}\text { Pregunta(s) } \\
\qquad N^{\circ}: \\
1,3,4,14\end{array}$ \\
\hline $\begin{array}{l}\text { Conocimiento: } \\
\text { - Distinguir hemisferios, círculo del Ecuador, trópicos, polos, } \\
\text { continentes y océanos del planeta en mapas y globos } \\
\text { terráqueos. } \\
\text { - Identificar y ubicar en mapas las principales zonas climáticas } \\
\text { del mundo, y dar ejemplos de distintos paisajes que pueden } \\
\text { encontrarse en estas zonas y de cómo las personas han } \\
\text { elaborado diferentes estrategias para habitarlos. } \\
\text { - Caracterizar el entorno geográfico de las civilizaciones } \\
\text { estudiadas, utilizando vocabulario geográfico adecuado } \\
\text { (continente, valle, montaña, océano, río, archipiélago, mares, } \\
\text { península, ciudad, construcciones y monumentos, entre otros). } \\
\text { Habilidades: } \\
\text { - Leer y comunicar información geográfica por medio de } \\
\text { distintas herramientas (planos, mapas, globos terráqueos y } \\
\text { diagramas). } \\
\text { Orientarse en el espacio, utilizando referencias, categorías de } \\
\text { ubicación relativa y puntos cardinales. }\end{array}$ & 3 Básico. & $\begin{array}{c}\text { Pregunta(s) } \\
\mathrm{N}^{\circ}: \\
5,6,7\end{array}$ \\
\hline
\end{tabular}




\begin{tabular}{|c|c|c|}
\hline Objetivos (conocimientos y habilidades) & $\begin{array}{c}\text { Nivel de } \\
\text { estudios } \\
\text { vinculado }\end{array}$ & $\begin{array}{c}\text { Preguntas } \\
\text { asociadas } \\
\text { a los objetivos }\end{array}$ \\
\hline $\begin{array}{l}\text { Conocimiento: } \\
\text { - Ubicar lugares en un mapa, utilizando coordenadas geográficas } \\
\text { como referencia (paralelos y meridianos). } \\
\text { Distinguir recursos naturales renovables y no renovables, } \\
\text { reconocer el carácter limitado de los recursos naturales y la } \\
\text { necesidad de cuidarlos, e identificar recursos presentes en } \\
\text { objetos y bienes cotidianos. } \\
\text { - Describir distintos paisajes del continente americano, } \\
\text { considerando climas, ríos, población, idiomas, países y grandes } \\
\text { ciudades, entre otros, y utilizando vocabulario geográfico } \\
\text { adecuado. } \\
\text { Reconocer y ubicar los principales recursos naturales de } \\
\text { América, considerando su distribución geográfica y su uso, y } \\
\text { reconociendo la importancia de cuidarlos en el marco de un } \\
\text { desarrollo sostenible. } \\
\text { Comparar, mediante la observación en imágenes, mapas y } \\
\text { otras fuentes, paisajes de su región con paisajes de América, } \\
\text { distinguiendo distintas formas de adaptación y transformación } \\
\text { de la población a su ambiente natural. } \\
\text { Habilidades: } \\
\text { Usar herramientas geográficas para ubicar y caracterizar } \\
\text { elementos del espacio geográfico, como paisajes, población y } \\
\text { recursos naturales. } \\
\text { Orientarse en el espacio, utilizando categorías de ubicación } \\
\text { absoluta (coordenadas geográficas) y relativa. }\end{array}$ & $4^{\circ}$ Básico & $\begin{array}{l}\text { Pregunta(s) } \\
\quad N^{\circ}: \\
5,6,7,21,23\end{array}$ \\
\hline $\begin{array}{l}\text { Conocimiento: } \\
\text { Caracterizar las grandes zonas de Chile y sus paisajes (Norte } \\
\text { Grande, Norte Chico, Zona Central, Zona Sur y Zona Austral), } \\
\text { considerando ubicación, clima temperatura y precipitaciones), } \\
\text { relieve, hidrografía, población y recursos naturales, entre otros. } \\
\text { - } \quad \text { Investigar, describir y ubicar los riesgos naturales que afectan } \\
\text { a su localidad, como sismos, maremotos, inundaciones, } \\
\text { derrumbes y volcanismo, e identificar formas en que la } \\
\text { comunidad puede protegerse. } \\
\text { - } \\
\text { Habilidades: } \\
\text { Usar herramientas geográficas para ubicar, caracterizar y } \\
\text { relacionar elementos del espacio geográfico, como regiones, } \\
\text { climas, paisajes, población, recursos y riesgos naturales. } \\
\text { - Orientarse en el espacio, utilizando categorías de ubicación } \\
\text { absoluta (coordenadas geográficas) y relativa. }\end{array}$ & $5^{\circ}$ Básico & $\begin{array}{c}\text { Pregunta(s) } \\
\mathrm{N}^{\circ}: \\
9,10,12,14 \\
15,20,22,24\end{array}$ \\
\hline
\end{tabular}


Continuación Cuadro $N^{\circ} 1$

\begin{tabular}{|c|c|c|}
\hline Objetivos (conocimientos y habilidades) & $\begin{array}{c}\text { Nivel de } \\
\text { estudios } \\
\text { vinculado }\end{array}$ & $\begin{array}{c}\text { Preguntas } \\
\text { asociadas } \\
\text { a los objetivos }\end{array}$ \\
\hline $\begin{array}{l}\text { Conocimiento: } \\
\text { Identificar elementos constitutivos del territorio nacional, } \\
\text { considerando la localización de Chile en el mundo y su carácter } \\
\text { tricontinental. } \\
\text { - Caracterizar geográficamente las regiones político- } \\
\text { administrativas del país, destacando los rasgos físicos } \\
\text { (como clima, relieve, hidrografía y vegetación) y humanos } \\
\text { (como volumen y distribución de la población y actividades } \\
\text { económicas). } \\
\text { - Explicar las principales características físicas, humanas y } \\
\text { económicas de su región y de su localidad. } \\
\text { Habilidades: } \\
\text { - Usar herramientas geográficas para ubicar, caracterizar y } \\
\text { relacionar elementos del espacio geográfico, como regiones, } \\
\text { climas, paisajes, población, recursos y riesgos naturales. }\end{array}$ & 6 Básico & $\begin{array}{l}\text { Pregunta(s) } \\
\quad \mathrm{N}^{\circ}: \\
11,13,16,17 \\
18,19,20\end{array}$ \\
\hline $\begin{array}{l}\text { Conocimiento: } \\
\text { - Caracterizar los componentes físicos del planeta y sus } \\
\text { dinámicas, incluyendo: } \\
\text { litósfera (actividad sísmica y volcánica) } \\
\text { atmósfera (clima, tiempo atmosférico y vientos) } \\
\text { hidrósfera (ciclo del agua) } \\
\text { Habilidades: } \\
\text { - } \quad \text { Formulación de relaciones entre distintos sistemas de la Tierra } \\
\text { - } \quad \text { Análisis del impacto de los procesos naturales y humanos. }\end{array}$ & $7^{\circ}$ Básico & $\begin{array}{l}\text { Pregunta(s) } \\
\qquad N^{\circ}: \\
8,9,10,20\end{array}$ \\
\hline
\end{tabular}

Del Cuadro $\mathrm{N}^{0} 1$ se puede resaltar los siguientes aspectos:

En el Nivel Básico 1 (NB1), que corresponde a 1ro. y 2do básico, resaltan las habilidades relativas a: (1) Localizar a Chile en mapamundis o globos terráqueos y distinguir diferentes representaciones de la Tierra. (2) Leer y comunicar información geográfica mediante distintas herramientas (planos, mapas, globos terráqueos y diagramas) y (3) Orientarse en el espacio, utilizando categorías de ubicación relativa (derecha, izquierda, lejos, cerca) y asociando referentes locales (cordiIlera, cerro, mar y otros) a los puntos cardinales.

En el Nivel Básico 2 (NB2), que corresponde a 3ro. y 4to. básico, las habilidades que caracterizan a la disciplina están relacionadas con: (1) Leer y comunicar información geográfica por medio de distintas herramientas (planos, mapas, globos terráqueos y diagramas). (2) Orientarse en el espacio, utilizando referencias, categorías de ubicación relativa y puntos cardinales. (3) Usar herramientas geográficas para ubicar y caracterizar elementos del espacio geográfico, como paisajes, 
población y recursos naturales y (4) Orientarse en el espacio, utilizando categorías de ubicación absoluta (coordenadas geográficas) y relativa.

Por su parte, en el Nivel Básico 3 (NB3), que corresponde al 5to. básico, se observan las siguientes habilidades: (1) Usar herramientas geográficas para ubicar, caracterizar y relacionar elementos del espacio geográfico, como regiones, climas, paisajes, población, recursos y riesgos naturales. (2) Orientarse en el espacio, utilizando categorías de ubicación absoluta (coordenadas geográficas) y relativa. (3) Formulación de relaciones entre distintos sistemas de la Tierra y (4) Análisis del impacto de los procesos naturales y humanos.

En Nivel Básico 4 (NB4), que corresponde al 6to. básico, resaltan las siguientes habilidades: (1) Usar herramientas geográficas para ubicar, caracterizar y relacionar elementos del espacio geográfico, como regiones, climas, paisajes, población, recursos y riesgos naturales.

Finalmente, en el Nivel Básico 5 (NB5), es decir el 7mo. Básico, se denotan: (1) Formulación de relaciones entre distintos sistemas de la Tierra. (2) Análisis del impacto de los procesos naturales y humanos.

En definitiva, serán estos campos cognitivos los que orientan la preparación del instrumento que se elabora, prueba, enjuicia por expertos y se prueba experimentalmente, previo a su aplicación en la muestra en estudio.

\section{Cuestionario de conocimientos y habilidades fundamentales}

\section{El cuestionario: sus antecedentes}

Como se indicó previamente, hubo una evaluación preliminar (Castillo y Jaime, 2012) que demostró un bajo nivel de conocimientos en la disciplina en estudiantes de 4to. año medio (Cuatro establecimientos de las Comunas de Rancagua y Valparaíso). Esta investigación amplió y diversificó la muestra de estudiantes evaluados con un número mayor de establecimientos y un área geográfica distinta a las anteriores, incluyendo a las Comunas de San Felipe y Los Andes, todos establecimientos accesibles al equipo de investigación, permitiendo definir una muestra del tipo no probabilística, intencionada, atendiendo que el objetivo comprende indagar sobre aspectos que eventualmente pueden ser comunes a los estudiantes ya investigados, que el objetivo no busca generalizaciones, que se trata de explorar un área de estudio de poco desarrollo hasta ahora y, además, en consideración a la accesibilidad a la muestra (Briones, 1996).

El Cuestionario de evaluación se elaboró teniendo como referente lo avanzado en la investigación de Castillo y Jaime (2012), la que fue inserta en calidad de estudio exploratorio dentro del plan de trabajo de la investigación. Derivado del análisis curricular realizado a los Programas de Estudio se elabora un cuestionario preliminar de cerca de 30 preguntas, las que son progresivamente mejoradas tras consultas sucesivas a académicos expertos del área de Geografía, arribando finalmente a definir 24 preguntas tipo selección múltiple repartidas entre las temáticas ya 
definidas tras el análisis curricular (MINEDUC, 2009)11. La Hoja de respuesta se estructuró en dos columnas, una que comprendía las opciones de respuesta sobre el contenido temático consultado o la aplicación de ese conocimiento a situaciones simuladas compuestas por cinco alternativas de respuesta. La segunda columna estaba destinada a expresar el grado de seguridad con que se entregaba cada una de las respuestas, la cual se divide a su vez en tres niveles de seguridad asociada a cada una de las respuestas dadas: "completamente seguro" (C.M.), "Medianamente seguro" (M.S.) e "Inseguro" (IN).

La información compilada se organizó en una base de datos, la que fue tratada estadísticamente empleando el programa SPSS-18, bajo criterios descriptivos e inferenciales (Howell, 1998).

\section{Análisis de validez y consistencia del instrumento}

El cuestionario fue aplicado a estudiantes de Cuarto año Medio de 11 establecimientos Municipales y Particulares Subvencionados de las Comunas de Valparaíso, Viña del Mar, Quilpué y San Felipe (total $n=428)$; hombres $(n=213)$ y mujeres $(n=215)$, de entre 15 y 18 años.

El primer análisis realizado comprendió el cálculo de la consistencia interna del instrumento, considerando que se trataba de su primera aplicación formal y dado el interés de los investigadores por validar e introducir mejoras en vistas a futuras aplicaciones. Se empleó el coeficiente "Alfa de Cronbach" el que arrojó un índice de ,715. Atendiendo que el instrumento comprende 24 preguntas, y siguiendo las indicaciones analíticas de Cronbach y Meehl (1955), se puede afirmar que alcanza un nivel aceptable y que da cuenta de una adecuada correlación interna entre sus elementos constitutivos (Osburn, 2000).

Teniendo en cuenta la confirmación de la calidad del instrumento, se realizó un segundo estudio de consistencia interna a partir de los puntajes obtenidos, primeramente, calculando la correlación interna de sus ítemes mediante un cálculo de relación entre cada ítem respecto del test completo (Índice de homogeneidad corregida). Este cálculo demostró que todos los ítemes denotan cifras cercanas al índice Alfa total del instrumento, exceptuando algunos que -eliminándoles- muestran aumento del índice total $=, 715$. En este sentido, la prueba correlación Total/ Elemento denota que eliminando ítemes como el $n^{\circ} 12$, el índice alfa aumenta $a=, 738$; empleando el mismo procedimiento con los Ítemes $4=, 727$ y $11=, 726$ se comprueba que muestran un aumento en la consistencia interna del instrumento. En definitiva, en términos de la estructura y consistencia del Cuestionario, se alcanza un nivel de consistencia que se ajusta a los parámetros validados por convención como aceptables (entre, 70 y ,90); respecto del conjunto de ítemes incluidos, algunos de ellos, identificados por las pruebas realizadas, debieran ser corregidos.

\section{Análisis de los resultados obtenidos}

Tratando los datos con enfoques distintos, siempre con el objeto de explorarles, el nivel promedio de aciertos (total) alcanzó al 32,83"\%; en el margen superior de acierto se ubican las Preguntas $n^{\circ} 24\left(N A^{12}=60,9\right)$ y $n^{\circ} 12(N A=55,4)$, en contrario, en el margen inferior los niveles más bajos

Ministerio de Educación, Gobierno de Chile. "Bases Curriculares y programas de estudio", ver en http://www.curriculumenlineamineduc.cl

12 $N A=$ Nivel de Acierto. 
de acierto lo tienen las Preguntas $n^{\circ} 9(N A=19 \%)$ y $n^{\circ} 7(N A=21.7)$. En general las demás preguntas se ubican en torno al nivel medio de aciertos $(32,83 \%)$ desde el punto de vista del contenido evaluado en estas preguntas "frontera", tres de ellas corresponde al ámbito de los conocimientos y solo una (P. $\left.n^{\circ} 24\right)$ al ámbito de la aplicación de esos conocimientos.

En cuanto a la omisión, el mayor índice de omisión $(I O)$ lo tienen las preguntas $n^{\circ} 5(I O=11), N^{\circ}$ $3, \mathrm{~N}^{\circ} 4, \mathrm{~N}^{\circ} 7$ y $\mathrm{N}^{\circ} 23(\mathrm{IO}=10)$, todas las cuales en general comprenden habilidades relacionadas con la aplicación de conocimientos; al contrario, las preguntas $N^{\circ} 1, N^{\circ} 16$ y $N^{\circ} 17$ denotan los más bajos índices de omisión $(\mathrm{IO}=2)$ y se corresponden con el dominio de conocimientos específicos.

Otro elemento evaluado describe el nivel de seguridad en relación con cada una de las respuestas dadas (relación directa entre respuesta y nivel de seguridad). Los resultados totales denotan una tendencia predominante desde la opción "Medianamente Seguro" (MS) a "Inseguro" (IN), excepto para las preguntas $\mathrm{N}^{\circ} 1, \mathrm{~N}^{\circ} 12, \mathrm{~N}^{\circ} 13, \mathrm{~N}^{\circ} 20$ y $\mathrm{N}^{\circ} 24$ que muestran preeminencia de la opción "Completa Seguridad" (CS). De este análisis se derivan dos informaciones interesantes. Primero, que existe coincidencia entre respuestas correctas en el caso de las Preguntas $N^{\circ} 12$ y $N^{\circ} 24$ y sus respectivos índices de seguridad (NA+CS). La segunda información atañe la notoria tendencia por la opción MS, lo cual se expresa sin una correlación directa con el nivel de acierto de cada pregunta, exceptuando las dos respuestas antes mencionadas.

Al emplear otro criterio, Agrupación de respuestas según los cursos en que se ubican los contenidos evaluados y el Porcentaje de logro, se produce la distribución que muestra el Cuadro $N^{\circ} 2$.

La dispersión entre el porcentaje mayor y menor alcanza a 11 puntos, siendo el más alto el que corresponde a segundo y tercer año, mismo nivel en que se ubica el porcentaje más bajo.

Cuadro $\mathrm{N}^{\circ} 2$

Niveles de logro según contenidos/cursos

\begin{tabular}{|l|r|}
\hline $\begin{array}{l}\text { Ubicación de contenidos } \\
\text { según cursos }\end{array}$ & \multicolumn{1}{|c|}{ Porcentaje de logro } \\
\hline Primero, Segundo y Tercero & $32 \%$ \\
\hline Segundo y Tercero & $38^{\prime \prime} \%$ (Más alto) \\
\hline Tercero & $27,3^{\prime \prime} \%$ (Más bajo) \\
\hline Cuarto & $28,4^{\prime \prime} \%$ \\
\hline Quinto & $35,3^{\prime \prime} \%$ \\
\hline Sexto & $31,7^{\prime \prime} \%$ \\
\hline Séptimo & $29,1^{\prime \prime} \%$ \\
\hline
\end{tabular}

Finalmente, al revisar los niveles de logro asociados a los ámbitos cognitivos estudiados se puede observar la siguiente distribución (Cuadro Nº 3 ) 
Cuadro $\mathrm{N}^{\circ} 3$

Niveles de logro según áreas del conocimiento

\begin{tabular}{|l|r|r|}
\hline & \multicolumn{1}{|c|}{ Conocimientos } & \multicolumn{2}{|c|}{ Habilidades } \\
\hline Porcentaje de aciertos & $32 \%$ & $30 \%$ \\
\hline Índice de seguridad promedio. & $28 \%$ & $25 \%$ \\
\hline
\end{tabular}

Del Cuadro $N^{\circ} 3$ se desprende lo siguiente:

- El porcentaje de conocimiento es aceptable y no se condice con el nivel de seguridad que un estudiante del nivel evaluado debiera tener al término de los ciclos de estudios.

- Una de la principales dibilidades que se detectó es que el alumno no tiene un manejo conciso de los temas espaciales, lo que limita el conocimiento de inicio de la educacion geográfica, es decir, la localizacion de hechos o fenómenos. Dicha situacion no cambia mucho en el año escolar 2015, dado que los textos escolares entregados por el Ministerio de Educacion (Cot y Morán, 2013) confunde las definiciones de los paralelos con el Ecuador. Este hecho limita la habilidad de leer y comunicar informacion geografica, situación que queda respaldada en la lectura e interpretacion de la gráfica (Climograma).

- En relación con el nivel de seguridad, este es igualmente bajo, lo cual queda evidenciado en preguntas que está relacionadas con las temáticas que refieren lo patrimonial.

- En las preguntas en donde se contextualizó el contenido (espacial y temporalmente), los resultados fueron óptimos; por ejemplo, aquellas preguntas que se relacionaron con el "27 $F^{\prime \prime}$, los alumnos respondieron en forma correcta, y su nivel de seguridad es coherente en resultados con el contenido evaluado.

El punto anterior permite inferir una conclusión en relación a los procesos de enseñanzaaprendizaje, la que se detalla en el apartado siguiente, y que apunta al hecho de que en aquellos contenidos que se contextualizan se logra generar un aprendizaje significativo, demostrando con ello que pasa a ser un contenido y una habilidad interiorizada y no memorizada.

\section{Conclusiones}

Antes de detallar las conclusiones que inspiran los resultados obtenidos, se puede afirmar que se confirma lo evaluado exploratoriamente (Castillo y Jaime, 2012) y, con ello, la hipótesis de trabajo que refiere el hecho de que los estudiantes evaluados denotarían falta de conocimiento básico en el área disciplinar de Geografía, expresado en un escaso manejo de información y un bajo desarrollo de habilidades estimadas esenciales en el área (Nivel Promedio de Aciertos Total= $\left.32,83^{\prime \prime} \%\right)$

Considerando el bajo indice de acierto que alcanzan los estudiantes, se puede adelantar algunas explicaciones de los resultados. Si se tiene a la vista el estudio curricular realizado al Programa, se constata el desmedro de la disciplina respecto de la Historia (constituyen un mismo Programa formativo); primeramente desde el punto de vista de la profundidad y desarrollo de la 
disciplina como tal y, en segundo término, desde la perspectiva del tiempo que se dispone para su desarrollo en el aula, es altamente problable que los docentes distribuyan el poco tiempo de que disponen en lo estrictamente básico de la asignatura, esto es la revisión de sus contenidos y el desarrollo de las habilidades fundamentales.

De acuerdo con lo anterior, se puede concluir, atendiendo la literatura consultada y la revisión de los conocimientos y habilidades básicas declarados en los Programas de Estudio, que el trabajo en clases responde a un desarrollo disciplinar centrado en la mera descripción de elementos físicos y en la entrega de información, sin establecer una relación con un trabajo interdisciplinario del curriculum. Se puede señalar que los objetivos fundamentales que desarrollan las habilidades y conocimientos básicos disciplinares para la educación básica y media (Programa de Estudio), no son consecuentes con el desarrollo de una geografía que ha definido como objeto de estudio el "espacio social" (Drucker, 2004), sin embargo estos elementos no están reflejados en el desarrollo de conocimientos y habilidades básicas del programa de estudios prescrito, manteniéndose solamente un contenido basado en una geografía descriptiva sin capacidad de análisis y sin una consecuencia con los demás contenidos desarrollados en las demás disciplinas sociales (Historia principalmente). Ejemplo de esto último se observa en los bajos porcentajes de asertividad alcanzados en respuestas que conciernen contenidos que todo estudiante debería manejar (nivel de acierto total $=32,8 \%$ ), como igualmente en los bajos niveles de seguridad en las respuestas manifestados por los alumnos (Tendencia a MS e IN).

Examinando las tres dimensiones mencionadas en la revisión teórica de la investigación (localización espacial y comprensión sistémica del espacio geográfico, habilidades de análisis del espacio geográfico, y valoración y actuación responsable con el espacio geográfico), y considerando el manejo conceptual que deben tener los estudiantes, se concluye en base a los niveles de "conocimiento" que el bajo nivel de seguridad manifestado por los estudiantes estaría demostrando que ellos, si bien responden los ítemes planteados, no manifiestan claridad conceptual en sus respuestas. El porcentaje de inseguridad (IN) sobrepasa el 30\% en 19 de las 24 preguntas; entre esas preguntas -los más altos índices- corresponden a la dimensión de "comprensión sistémica del espacio geográfico" con P. $n^{\circ} 7, I N=42,5$ y P. $n^{\circ} 14, I N=35,7$. Para contrastar este resultado aparece la pregunta que obtiene el mayor porcentaje de frecuencia de respuestas correctas, pregunta 1: "Si queremos complementar nuestra percepción del espacio cotidiano. ¿Con que tipo de representación cartográfica debo hacerlo?" (RC ${ }^{13}$ : un plano), lo que podría demostrar que los estudiantes reconocen que los mapas y los planos son formas de representar lugares, utilizando un lenguaje geográfico adecuado, discriminando entre las representaciones cartográficas y su utilidad.

\section{Proyecciones}

Al comienzo se señaló que la realización del proyecto de evaluación que se informa ha sido un imperativo que surge desde la condición nuestra de formadores de futuros profesores (e investigadores); un "deber" que emerge además de compartir el área disciplinaria y constatar en el día a día de las aulas de los establecimientos educacionales como la geografía resulta accesoria en todo el proceso formativo de los estudiantes, incorporada como un contenido complementario 
luego de que se han tratado otros temas (y si queda tiempo) y además, lo que resulta más preoupante, abordando mínimos conocimientos, con una didáctica inapropiada, con recursos escasos, descontextualizada y con poca claridad teórica.

Ciertamente que los resultados obtenidos se pueden emplear como un argumento que denote la situación de la disciplina en el currículo oficial y, desde ahí, poder contribuir a iniciar un proceso de reflexión y de revisión de las estrategias didácticas y evaluativas docentes en los establecimientos educacionales. Parece necesario reconsiderar el valor y el aporte de la disciplina a la formación de los estudiantes, como área del conocimiento que les entrega una visión que centra y da sentido a la relación de ellos con el medio, entendiendo que cada sujeto es construido y es constructor del espacio que habita y, por tanto, se trata de un ser activo, dinámico y protagonista del medio en donde está incerto.

Una muestra más amplia de estudiantes permitiría verificar con mayor consistencia la realidad que se enfrenta, desde el punto de vista de sus resultados, focalizando con más amplitud y precisión los problemas que aquejan el aprendizaje en la disciplina. En este mismo sentido es necesario introducir mejoras en el instrumento, en particular a nivel de algunos de los reactivos que propone, precisa de incorporar nuevas preguntas de modo de profundizar y mejorar el estudio de los campos de conocimientos y habilidades ya explorados, pero sobre todo se requiere ampliar el estudio al ámbito valórico-actitudinal.

\section{Corolario}

Aceptando que el modelo curricular de un país es el producto que resulta de un acuerdo sobre nociones fundamentales en todas las disciplinas de estudio, compartido entre el mundo científico-académico y las autoridades educacionales, éste cobra valor en cuanto deviene referente que orienta y guía los procesos de planificación que desarrollan los profesores pero, mucho más alla de eso, las orientaciones del Marco Curricular Nacional se transforman en desafíos que interpelan a los docentes en su trabajo cotidiano, desafíos que establecen criterios y orientaciones para "la actuación pedagógica" del aula. En esa perspectiva, en la disciplina geografica, los lineamientos oficiales expresan objetivos fundamentales como el siguiente:

"El sector Historia, Geografia y Ciencias Sociales tiene por proposito desarrollar en alumnos y alumnos conocimientos, habilidades y disposiciones que le permitan estructurar una comprension del entorno social y su devenir, y les oriente a actuar critica y responsablemente en la sociedad, sobre la base de los principios de solidaridad, pluralismo, cuidado del medioambiente, valoración del medio ambiente y de la identidad nacional. El curriculum del sector promueve aprendizajes que le signifiquen un aporte para enfrentar los desafios que le impone su existencia en un mundo que cambia aceleradamente y es cada vez mas complejo e interconectado" (MINEDUC, 2009: 195)

El objetivo citado inspira una crítica sobre el contenido que se enseña en la disciplina de Geografía, el que adquiere en el trabajo didáctico una fisonomía fragmentada y desarticulada respecto de otras disciplinas del área de la Historia y las ciencias sociales, lo que dificulta de una parte la transposición didáctica para el docente que enseña y, de otra parte, dificulta el aprendizaje significativo del sujeto que aprende. 
Tanto las condiciones de que disponen los estudiantes en la actualidad para aprender, como las formas y métodos que emplean para hacerlo, y las posibilidades y recursos con que cuentan los docentes en la actualidad para enseñar, impone el desafío de establecer una congruencia entre los nuevos enfoques epistémicos de la disciplina y de la enseñanza de la geografía. Desde esta perspectiva se hace imperioso pasar desde un enfoque técnico de la didáctica ("recetas focalizadas en las tareas") a un enfoque que exprese una didáctica contextual centrada en la comprensión de la experiencia del sujeto en relación con sus espacios (sujeto como creador y recreado de espacios); una didáctica en la que el espacio y el territorio dejan de ser -como señala Margalef (2005: 10) - "...un lugar en el que se aprende para ser un lugar del que se aprende".

\section{Referencias bibliográficas}

AUSUBEL, D. Psicología Educativa. Un punto de vista cognoscitivo. México: Trillas, 1987.

BARDIN, L. El análisis de contenido. Madrid: Akal, 1996.

BRIONES, G. Metodología de la investigación cuantitativa en las ciencias sociales. Bogotá: ICFES, 1996.

CASTILLO, G. y JAIME, J. Evaluación de los conocimientos de Geografía en alumnos (as) de 8vo. Básico y 4to. Año Medio de Establecimientos particulares subvencionados y Municipales pertenecientes a las ciudades de Rancagua y Valparaíso. Valparaíso: Tesis de grado de Pedagogía en Historia y Geografía, Universidad de Playa Ancha, 2012.

COLL, C.; POZO, J.I.; SARABIA, B. y VALLS, E. Los contenidos de la reforma: Enseñanza y aprendizaje de conceptos, procedimientos y actitudes. Madrid: Santillana, 1994.

CRONBACH, L. \& MEEHL, P. Construct validity in psychological tests. Psychological Bulletin, 1955, Vol. 52, N4, p. 281-302.

COT, N. y MORÁN, M.J. Historia, Geografía y Ciencias Sociales, Cuarto Año Básico. Texto del estudiante. Santiago de Chile: Norma, 2013.

DE CAMILLONI, A.W. De herencias, deudas y legados. Una introducción a las corrientes actuales de la didáctica. En: DE CAMILLONI, A.W.; DAVINI, M.C.; EDELSTEIN, G.; LITWIN, E.; SOUTO, M. y BARCO, S. Corrientes didácticas contemporáneas. Buenos Aires: Paidós, 1996.

DELORS, J. La educación encierra un tesoro. Paris: UNESCO, 1996.

DRUCKER, P. La sociedad postcapitalista. Buenos Aires: Norma, 2004.

GALLASTEGUI, J. y GALEA, J. Espacios para una Geografía social, humanista y crítica. Valparaíso: Editorial Facultad de Humanidades, 2009.

GALLASTEGUI, J.; GALEA, J. y ROJAS, I. Introducción a la Teoría Geográfica. Valparaíso: Editorial Facultad de Humanidades, 2014. 
GIMENO, J. y PÉREZ, A. (editores). La enseñanza: su teoría y su práctica. Madrid: Akal, 1983.

HIERNAUX, D. y LINDON, A. Introducción. La Geografía Humana: un camino a recorrer. En: HIERNAUX, D. y LINDON, A. (directores). Tratado de geografía humana. Barcelona: Anthropos-UAM, 2006, p. 7-22.

HOWELL, D. Méthodes statistiques en sciences humaines. Bruselas: De Boeck, 1998.

KEMMIS, S. El curriculum: más allá de la teoría de la reproducción. Madrid: Morata, 1993.

KRIPPENDORF, K. Metodología de análisis de contenido. Barcelona: Paidós, 1990.

LICERAS, A. Tratamiento de las Dificultades de aprendizaje en Ciencias Sociales. Granada: Grupo Editorial Universitario, 2000.

LINDON, A. De las geografías constructivistas a las narrativas de vida espaciales como metodologías geográficas cualitativas. Revista da ANPEGE, 2008, N 4, p. $3-27$.

MARGALEF, L. Innovar desde dentro: transformar la enseñanza más allá de la convergencia europea. Revista Iberoaméricana de Educación, 2005, Vol. 37, № 3, p. 1-12.

MÉNDEZ, R. El espacio de la geografía humana. En: PUYOL, R.; ESTEBANEZ, J. y MENDEZ, R. (coordinadores). Geografía humana. Madrid: Colección Cátedra Geografía, 1995, capítulo 1.

MÉNDEZ, R. Trayectoria recientes de la Geografía algunos problemas y potencialidades para su enseñanza. Revista Huellas, 2008, №12, p. 128-155.

MILES, M. \& HUBERMAN, M. Analyse des données qualitatives. Bruselas: De Boeck, 2003.

MINISTERIO DE EDUCACIÓN (MINEDUC). Objetivos Fundamentales y Contenidos Mínimos Obligatorios de la Educación Básica y Media. Actualización 2009. Santiago de Chile: Ministerio de Educación, 2009.

MUCCHIELLI, R. L'analyse de contenu: Des documents et des communications. París: ESF Editeur, 2006.

NOVAK, J. Conocimiento y aprendizaje. Los mapas conceptuales como herramientas facilitadoras para escuelas y empresas. Madrid: Alianza, 1998.

OSBURN, H.G. Coefficient alpha and related internal consistency reliability coefficients. Psychological Methods, 2000, Vol. 5, №3, p. 343-355.

PRATS, J. Curriculo escolar y formación de Profesores. En: Didáctica de las ciencias Sociales. Jaén: Editorial Universidad de Jaén, 2011.

RODRÍGUEZ, E. Geografía Conceptual. Enseñanza y aprendizaje de la geografía en la educación básica secundaria. Cali: Geopaideia, 2010. 
SANTOS, M. La naturaleza del espacio. Técnica y tiempo. Razón y emoción. Barcelona: Ariel, 2000.

UNIVERSIDAD DE PLAYA ANCHA (UPLA). Modelo Educativo. Valparaíso: Vicerrectoría académica, 2013a. Disponible en Internet: http://www.upla.cl/inicio/2012_0327_modelo_educativo.pdf

UNIVERSIDAD DE PLAYA ANCHA (UPLA). Convenio de Desempeño UPLA - MINEDUC 2013-2015. 2013b. Disponible en Internet: http://www.upla.cl/convenio/ 